\title{
Detection of the Hematopoietic Stem and Progenitor Cell Marker CD133 during Angiogenesis in Three-Dimensional Collagen Gel Culture
}

\author{
Masumi Akita, ${ }^{1}$ Kayoko Tanaka, ${ }^{1}$ Sachiko Matsumoto, ${ }^{1}$ \\ Kumiko Komatsu, ${ }^{1}$ and Keiko Fujita ${ }^{2}$ \\ ${ }^{1}$ Division of Morphological Science, Biomedical Research Center, Saitama Medical University, 38 Moroyama, \\ Iruma-gun, Saitama 350-0495, Japan \\ ${ }^{2}$ Department of Anatomy, Saitama Medical University, 38 Moroyama, Iruma-gun, Saitama 350-0495, Japan \\ Correspondence should be addressed to Masumi Akita; makita@saitama-med.ac.jp
}

Received 6 December 2012; Revised 9 May 2013; Accepted 3 June 2013

Academic Editor: Kenneth Boheler

Copyright (c) 2013 Masumi Akita et al. This is an open access article distributed under the Creative Commons Attribution License, which permits unrestricted use, distribution, and reproduction in any medium, provided the original work is properly cited.

We detected the hematopoietic stem and progenitor cell marker CD133 using immunogold labeling during angiogenesis in a threedimensional collagen gel culture. CD133-positive cells were present in capillary tubes newly formed from aortic explants in vitro. The CD133-positive cell population had the capacity to form capillary tubes. Lovastatin strongly inhibited cell migration from aortic explants and caused the degradation of the capillary tubes. The present study provides insight into the function of CD133 during angiogenesis as well as an explanation for the antiangiogenic effect of statins.

\section{Introduction}

CD133 was first isolated and cloned in 1997. CD133 expression was originally observed in hematopoietic stem and progenitor cells using a monoclonal antibody called AC133 [1] and neuroepithelial cells using a monoclonal antibody called prominin [2]. Gehling et al. [3] reported that the CD133-positive cell population consists of progenitor and stem cells that not only have hematopoietic potential but also have the capacity to differentiate into endothelial cells. Invernici et al. [4] reported that human fetal aorta contains vascular progenitor cells capable of inducing vasculogenesis and angiogenesis. Barcelos et al. [5] reported that human CD133 progenitor cells promote the healing of diabetic ischemic ulcers by paracrine stimulation of angiogenesis and activation of Wnt signaling.

To grow, solid tumors require a blood supply. They recruit new blood vessels mainly by inducing the sprouting of endothelial cells from external vessels. Recent research in tumor biology shows that, in addition to recruiting vessels from outside the tumor, brain tumors produce endothelial cells for vessel formation within the tumor [6]. Wang et al. [7] reported that a glioblastoma cell population (CD144 and CD133 double positive) differentiated into endothelial cells and formed intracellular vacuolar structures in collagen gel. However, the biological function of CD133 in angiogenesis remains largely unknown.

For in vitro studies of angiogenesis, several culture techniques using matrix structures have been developed, including fibrin and collagen gels [8], Matrigel, collagen, fibrin, and plasma clots [9]. Collagen gel culture has been used widely and effectively for analyzing the biological process of angiogenesis [10-13]. Using a three-dimensional (3D) collagen gel culture, we have conducted electron microscopic studies [14, 15] and immunohistochemical studies of fibroblast growth factor- (FGF-) 2 and FGF-9 [16]. Additionally, we have used $3 \mathrm{D}$ collagen gel cultures to test angiogenic and anti-angiogenic agents (TNF- $\alpha$ and thalidomide) $[17,18]$ to study vascular injury after laser microdissection [19] and for profiling of DNA microarray gene expression during angiogenesis [20]. The $3 \mathrm{D}$ collagen gel culture system provides a simple and rapid method to analyze angiogenesis. 
In the present study, we detected CD133 by immunogold labeling during angiogenesis in the $3 \mathrm{D}$ collagen gel culture system. Here, we show that CD133-positive cell population has a capacity to form capillary tubes.

\section{Materials and Methods}

2.1. Animals. ICR mice (male, 1 month old, $n=5$; CLEA Japan, Inc.) and Wistar rats (male, 2 months old, $n=3$; CLEA Japan, Inc.) were used for the experiment. The mice and rats were maintained according to the guidelines on the care and use of laboratory animals established by Saitama Medical University. These experiments were approved by the Animal Research Committee of Saitama Medical University.

2.2. Collagen Gel Culture of Mouse and Rat Aortae. The collagen culture technique used in the present study was modified from our previous technique $[14,15]$. Thoracic aortae were obtained from mice and rats. Under a stereoscopic microscope, fibroadipose tissue and blood were removed from the aortae. The thoracic aortae were then serially cross-sectioned into $\sim 2 \mathrm{~mm}$ rings. Four pieces were placed at the bottom of each tissue culture dish ( $35 \mathrm{~mm} ; n=25)$, overlaid with an even layer of reconstituted collagen solution ( $0.3 \%$ Cellmatrix type IA, Nitta Gelatin, Tokyo, Japan), and allowed to gel at $37^{\circ} \mathrm{C}$ for approximately $10 \mathrm{~min}$. After the gels formed, they were overlaid with Ham's F-12 medium (Invitrogen Corp., Carlsbad, CA, USA), containing 20\% fetal bovine serum (FBS), 1\% nonessential amino acids, 100 units/mL penicillin, and $100 \mathrm{mg} / \mathrm{mL}$ streptomycin (Invitrogen Corp., Carlsbad, CA, USA), and cultured for 14 days in an incubator ( $95 \%$ air $/ 5 \% \mathrm{CO}_{2}$ ). The medium was replaced three times a week starting from day 3. Capillary tube formation was observed using a phase contrast microscope during the culture period. These experiments were performed three times.

2.3. Phase-Contrast Microscopy and Time-Lapse Imaging. Standard phase-contrast images were collected by using a phase-contrast inverted microscope (Nikon TE2000, Japan) and a CCD camera (ORCA-ER, Hamamatsu Photonics, Japan). For time-lapse experiments, the aortic rings were cultured as described above. Cells that grew out from the aortic rings were visualized using a phase-contrast inverted microscope equipped with a stage that was preheated to $37^{\circ} \mathrm{C}$. The cells were maintained under $5 \% \mathrm{CO}_{2}$ in a culture chamber during image acquisition, and images were recorded at $5 \mathrm{~min}$ intervals using an Aquacosmos imaging system (Hamamatsu Photonics, Japan).

2.4. Transmission Electron Microscopy. The cultured aortic rings were fixed in $0.1 \mathrm{M}$ phosphate buffer $(\mathrm{pH}$ 7.2) containing $2.5 \%$ glutaraldehyde for 1 hour and then fixed in $0.1 \mathrm{M}$ phosphate buffer ( $\mathrm{pH} 7.2$ ) containing $1 \% \mathrm{OsO}_{4}$ for 1 hour. The rings were dehydrated in graded ethanol, embedded in epoxy resin, cut into ultrathin sections, and stained with uranyl acetate and lead citrate. The stained ultrathin sections were observed under a transmission electron microscope (JEM1010, Tokyo, Japan).
2.5. Rhodamine-Phalloidin and Lectin Histochemistry. After fixation in $4 \%$ paraformaldehyde/PBS, the cultured aortic rings were stained with rhodamine-phalloidin (Invitrogen Corp., Carlsbad, CA, USA) to determine the presence of Factin, and FITC-conjugated endothelial-cell-specific tomato lectin (Lycopersicon esculentum, EY Labo, CA, USA), which selectively binds to fucose residues that are present on the endothelial cell surface, was used to label endothelial cells [21].

2.6. Immunohistochemical Detection of CD133. The cultured aortic rings were fixed in $4 \%$ paraformaldehyde/PBS. For the detection of CD133 on the cell surface, the rings were incubated overnight with CD133 antibody (rabbit polyclonal, Abcam, Tokyo, Japan) after treatment with 1\% skim milk/PBS for $30 \mathrm{~min}$, and then they were incubated with Alexa Fluor 488- and Nanogold (1.4 nm)-conjugated goat anti-rabbit IgG (Nanoprobes, Inc., Yaphank, NY) for 1 hour. The Nanogold signal was enhanced using GoldEnhance EM (Nanoprobes) at room temperature for 3-5 min for electron microscopy and 20-25 min for light microscopy.

2.7. Effect of Lovastatin (Mevinolin) on Angiogenesis. Before and after tube formation, the effect of lovastatin (mevinolin from Aspergillus sp.) was tested. Lovastatin (mevinolin, M2147) supplied by Sigma has an empirical formula of $\mathrm{C}_{24} \mathrm{H}_{36} \mathrm{O}_{5}$ and is 2-methyl-1,2,3,7,8,8a-hexahydro-3,7-dimethyl-8-[2-(tetrahydro-4-hydroxy-6-oxo-2H-pyran-2-yl)-ethyl]-1-naphthalenyl ester butanoic acid. It is a white crystalline powder that is insoluble in water. A stock solution was prepared by dissolving it in $100 \%$ ethanol at a concentration of $12.3 \mathrm{mM}$ [22]. Two different sets of experiments were designed as follows.

Before Tube Formation. Rat aortic rings were cultured in $35 \mathrm{~mm}$ dishes as described above. After 24 hours, aortic rings were cultured with $12.3 \mathrm{mM}$ lovastatin or without lovastatin. Cultures were maintained at $37^{\circ} \mathrm{C}$ under $5 \% \mathrm{CO}_{2}$ in a humidified incubator.

After Tube Formation. Rat aortic rings were cultured in $35 \mathrm{~mm}$ dishes as described above. After 10 days, lovastatin was added to the culture medium. Cultures were maintained at $37^{\circ} \mathrm{C}$ under $5 \% \mathrm{CO}_{2}$ in a humidified incubator.

2.8. Endothelial Cell Scraping. In a separate set of experiments, the trimmed thoracic aorta was cultured as follows. To visualise the intimal surface directly, the thoracic aorta was everted with a procedure that sequestered the adventitial cells and possible remnant microvessels of periaortic soft tissue inside the aortic tube [23]. The endothelial cells were scraped from the everted aorta with a sterile cotton swab. The everted aorta with/without endothelium was cut into small pieces and cultured in the same manner as previously described, and followed by $10 \%$ formalin fixation and Giemsa staining. 


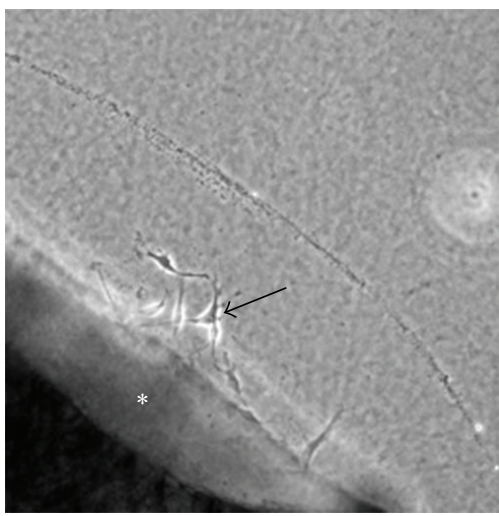

(a)

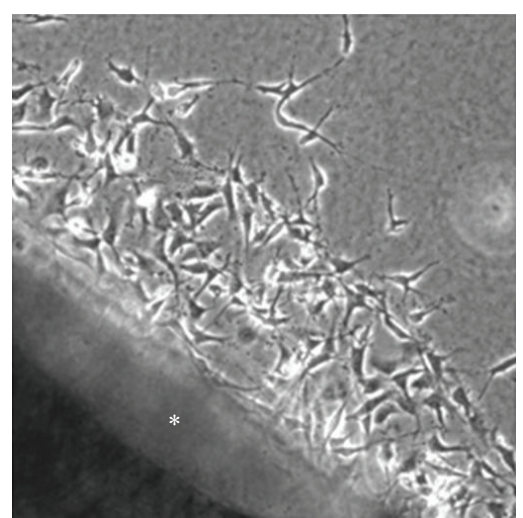

(b)

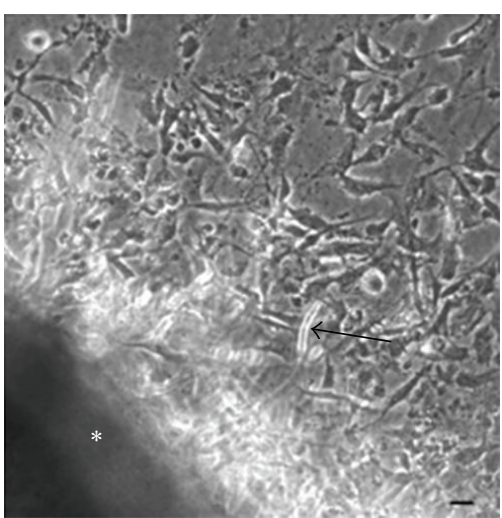

(c)

Figure 1: Phase-contrast microscopy. (a) After 2 days of cultivation, phase-contrast microscopy revealed fibroblastic cells (arrow) outgrown from a mouse aortic explant $(*)$ into a three-dimensional collagen gel. (b) After 5 days of cultivation, phase-contrast microscopy showed numerous fibroblastic cells outgrown from an aortic explant $(*)$. (c) After 7 days of cultivation, phase-contrast microscopy showed a tubular structure protruding (arrow) from an aortic explant $(*)$ into a three-dimensional collagen gel. (a), (b), and (c): Scale bar $=20 \mu \mathrm{m}$.
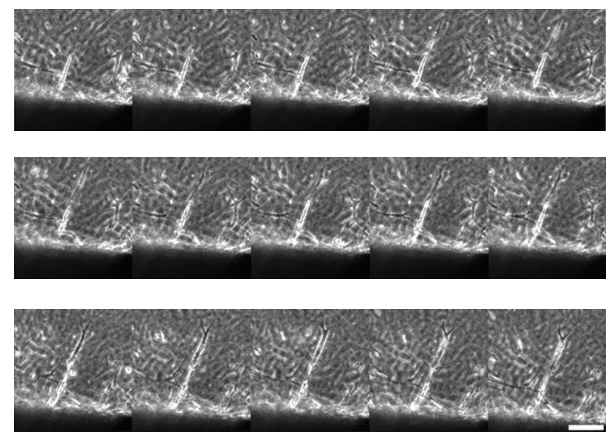

FIGURE 2: Time-lapse imaging of additional sprouts emerging and extending in a sequential manner from the leading edges of a newly formed capillary tube from the mouse aortic explant. Selected sequence from a time-lapse movie focusing on a single sprout. Note the protrusion of lamellipodia and continued migration of the leading cell. Scale bar $=50 \mu \mathrm{m}$.

\section{Results}

\subsection{Capillary Tube Formation}

3.1.1. Phase-Contrast Microscopy. Microscope examination after as little as 2 days of culture revealed the presence of migrating cells proximal to the aortic ring in the collagen gel. These cells were spindle-shaped and their longitudinal axes were radially orientated toward the stump of the aortic ring (Figures 1(a) and 1(b)). After a 7-day culture period, capillary sprouts were recognizable (Figure 1(c)), although lumen formation was not observed in these early capillary structures.

3.2. Time-Lapse Imaging of Capillary Tube Formation. Timelapse imaging was used to visualize the dynamic process of capillary tube formation from the aortic ring. Additional sprouts emerged and extended in a sequential manner from the leading edges of newly formed capillary tubes (Figure 2).

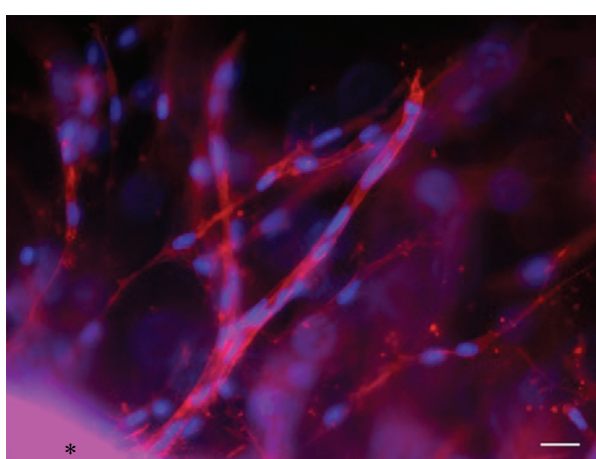

(a)

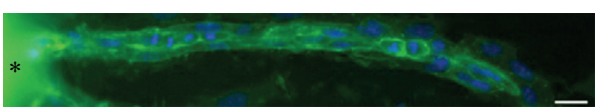

(b)

FIGURE 3: Rhodamine-phalloidin and lectin histochemistry. (a) After 11 days of cultivation, fluorescence microscopy showed capillary tubes that are stained with rhodamine-phalloidin for F-actin (purple-red) and 4',6-diamidino-2-phenylindole (DAPI) nucleic acid stain (blue). The asterisk (*) shows the mouse aortic explant. Scale bar $=20 \mu \mathrm{m}$. (b) After 11 days of cultivation, the capillary tubes are strongly positive for FITC-conjugated endothelial-cell-specific tomato lectin staining (yellowish-green) and DAPI nucleic acid stain (blue). The asterisk $(*)$ shows the aortic explant. Scale bar $=20 \mu \mathrm{m}$.

3.3. Rhodamine-Phalloidin and Lectin Histochemistry. After 10 to 14 days in culture, elongated capillary tubes with branches were observed. Capillary tubes in the collagen gels were observed by rhodamine-phalloidin staining (Figure 3(a)). Capillary tubes that formed in the collagen gels were also strongly positive for tomato lectin (Figure 3(b)).

3.4. Transmission Electron Microscopy. As demonstrated by cross-sectioning, endothelial cells of the capillary tubes formed tight contacts with each other, and pericyte-like cells 


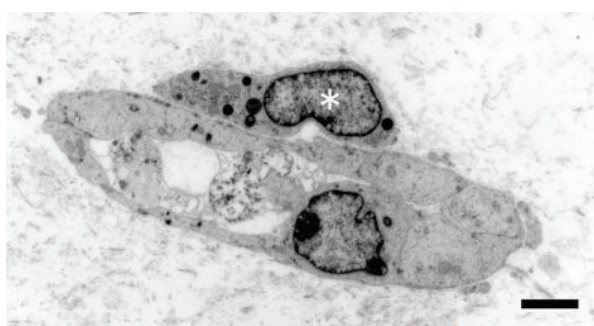

(a)

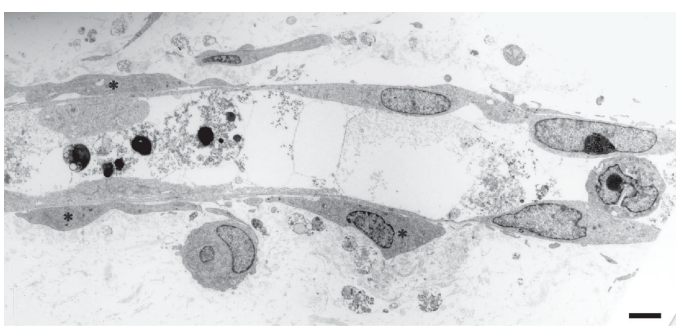

(b)

FIGURE 4: Transmission electron microscopy. (a) Electron microphotograph of a newly formed capillary tube from the mouse aortic explant in the collagen gel. A cross-section shows a capillary tube with a lumen that contains cell debris. Pericyte-like cells $(*)$ surround the tube. The edges of the cells are in contact with each other. Scale bar $=2 \mu \mathrm{m}$. (b) Electron microphotograph of a longitudinal section of a capillary tube. Pericyte-like cells $(*)$ surround the tube. Scale bar $=2 \mu \mathrm{m}$.

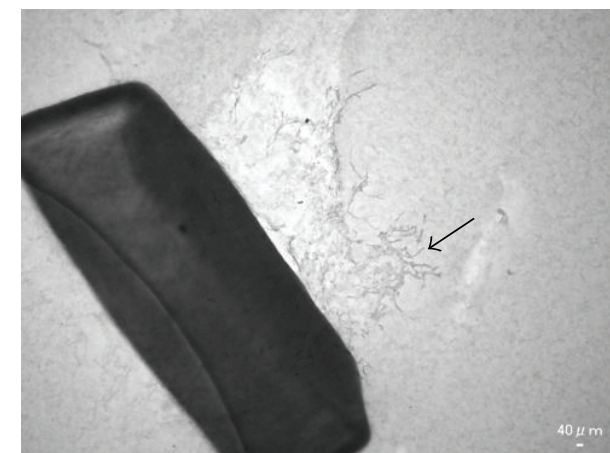

(a)

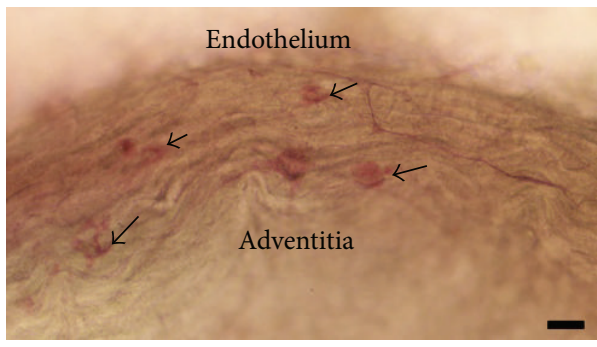

(c)

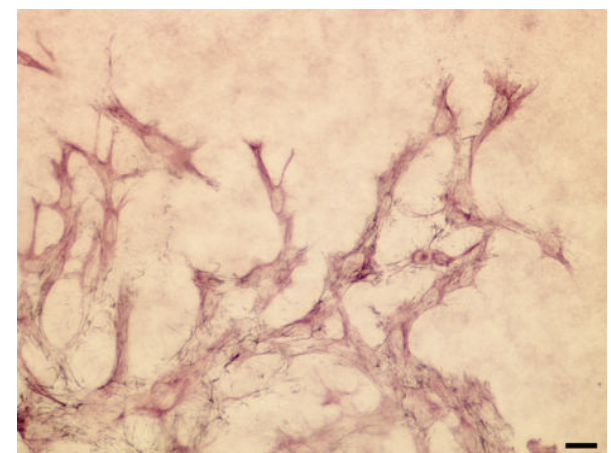

(b)

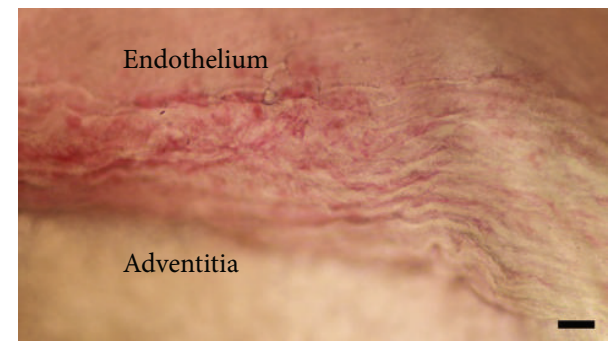

(d)

FIGURE 5: Immunohistochemistry of CD133. (a) In the early stages of culture (4-day culture), CD133-positive cells (arrow) were detected among cells migrating from the rat aortic explant. (b) Enlarged image of migrating CD133-positive cells. Scale bar $=20 \mu \mathrm{m}$. (c) CD133 expression (arrows) was found in the smooth muscle layer and near the adventitia. Scale bar $=20 \mu \mathrm{m}$. (d) CD133 expression was also found in the endothelium and the smooth muscle layer. Scale bar $=20 \mu \mathrm{m}$.

were present on the outside of the endothelial cells (Figure 4(a)). The endothelial cells did not show any pores or gaps. Typical gap junctions and tight junctions were not observed. Cell organelles were present in large numbers, particularly in the thicker endothelial cells. Longitudinal sectioning revealed that endothelial cells and pericyte-like cells made close contact (Figure 4(b)).

3.5. Immunohistochemical Detection of CD133. In the early stages of culture, CD133-positive cells were detected among cells migrating from the aortic ring (Figures 5(a) and 5(b)). CD133 expression was also found within the aortic wall (Figures 5(c) and 5(d)). In the later stages of culture, capillary tubes formed. CD133-positive cells were present in a tubelike pattern (Figures 6(a) and 6(b)), CD133 expression was found in both the tip and stalk regions. The leading edge of the capillary tube was strongly positive for CD133 (Figure 6(c)). Electron microscopic observation revealed CD133 expression in cells located on the bottom of the collagen gels (Figure 7).

\subsection{Effect of Lovastatin (Mevinolin) on Angiogenesis}

Before Tube Formation. When aortic rings were cultured with lovastatin, cell migration was strongly inhibited relative to the control (Figures 8(a) and 8(b)).

After Tube Formation. Lovastatin treatment induced the degradation of newly formed capillary tubes (Figures 9(a), 9(b), and 9(c)). Cell-cell adhesion was diminished, and the morphology of many CD133-positive cells changed to an oval 


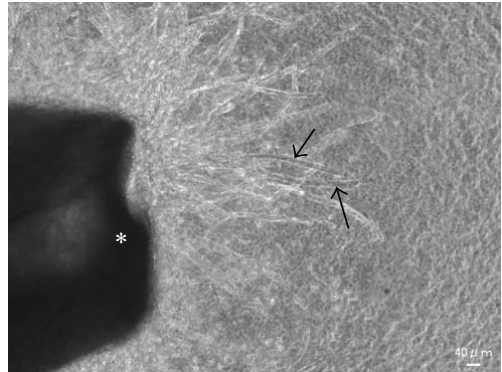

(a)

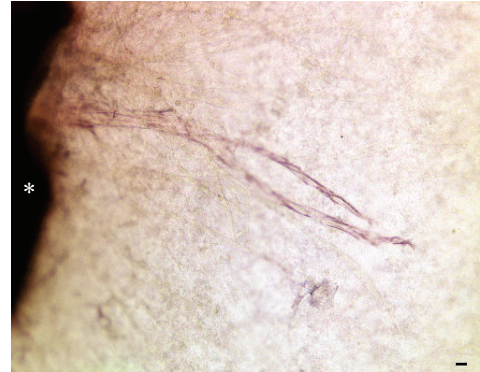

(b)

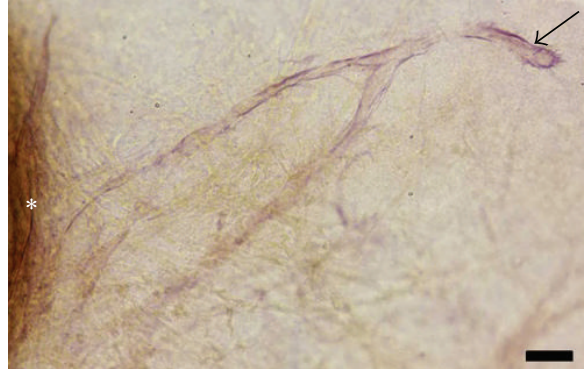

(c)

FIGURE 6: CD133 immunoreactivity in capillary tubes. (a) In the later stages of culture (9-day culture), capillary tubes formed. Phase-contrast microscopy showed that many tubes were formed from the rat aortic explant. Arrows indicate CD133-positive tubes among CD133-negative tubes. Rat aortic explant $(*)$. (b) CD133-positive cells were present in a tubular pattern. Photograph showing CD133-positive tubes indicated by arrows in (a). Rat aortic explant $(*)$. Scale bar $=20 \mu \mathrm{m}$. (c) CD133 expression was clearly found in the tip region (arrow) of the tube rather than the stalk region near the rat aortic explant $(*)$. Scale bar $=20 \mu \mathrm{m}$.

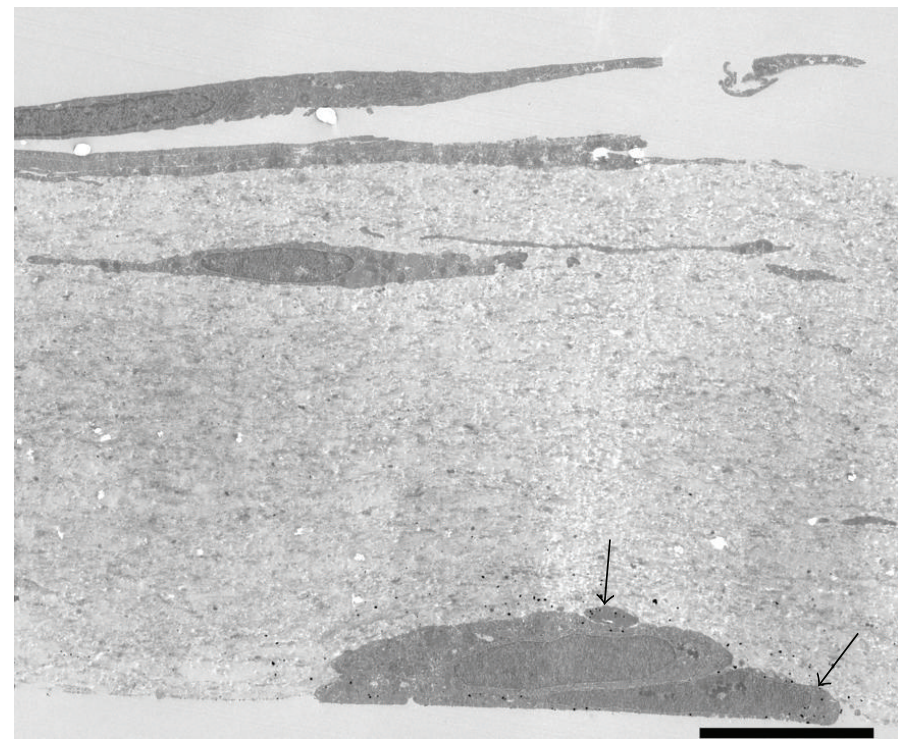

(a)

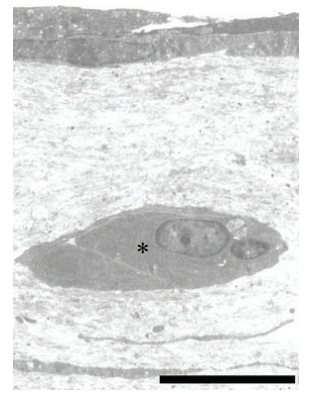

(b)

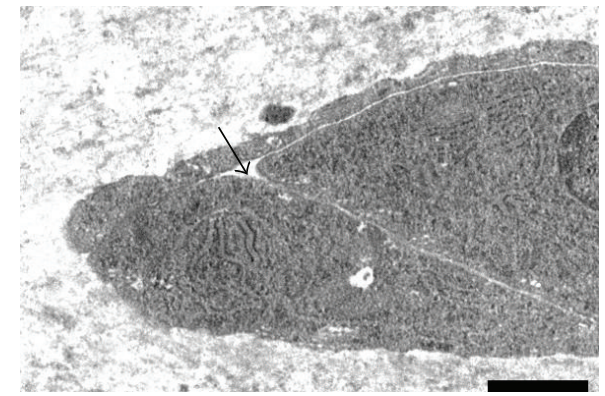

(c)

FIGURE 7: Immunoelectron microscopy of CD133. (a) At the bottom side of the collagen gels, CD133-positive cells made contact with each other (arrows). Cell organelles were sparse in the cells. Scale bar $=5 \mu \mathrm{m}$. (b) CD133-negative cells made contact with each other in the middle layer of the collagen gels $(*)$. Scale bar $=5 \mu \mathrm{m}$. (c) Enlargement of (b) $(*)$. These cells formed intercellular vacuolar structures (arrow). Cell organelles, such as the rough endoplasmic reticulum, were rich in these cells. Scale bar $=1 \mu \mathrm{m}$. 


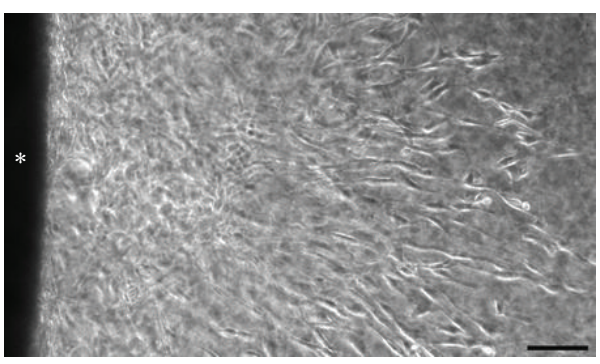

(a)

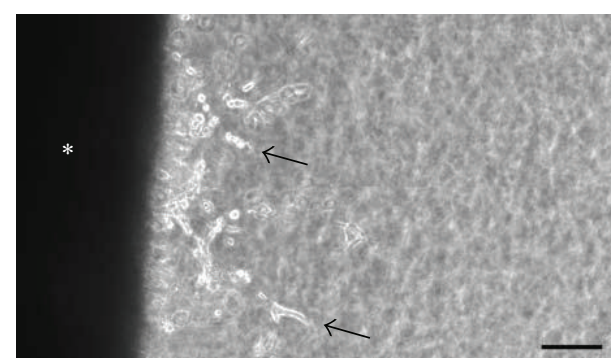

(b)

FIGURE 8: Effect of lovastatin (mevinolin) before tube formation. (a)-(b) When rat aortic rings were cultured with lovastatin, cell migration (arrows) was strongly inhibited relative to the control. (a) $=$ control, (b) $=$ lovastatin, aortic explant $(*)$. Scale bar $=100 \mu \mathrm{m}$.

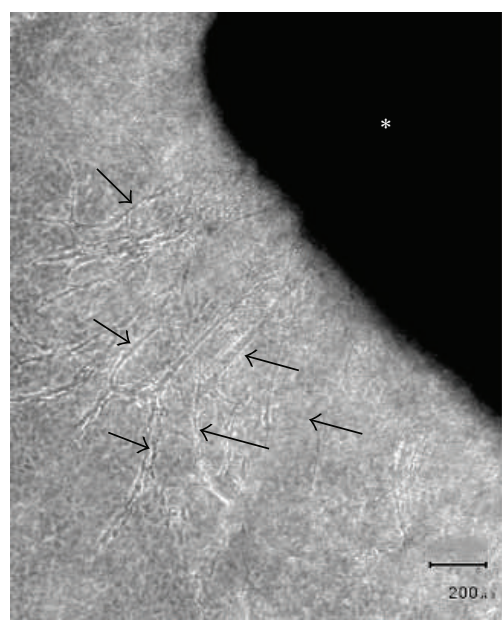

(a)

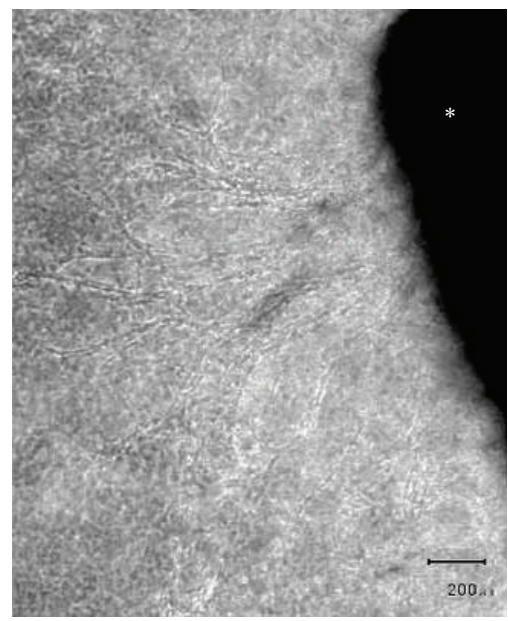

(b)

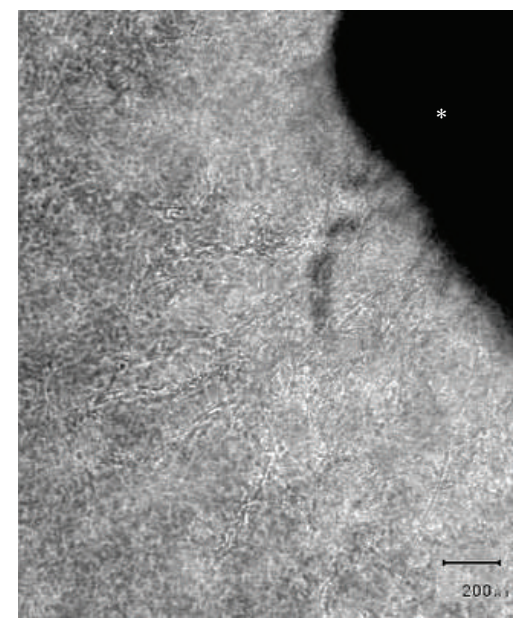

(c)

FIGURE 9: Effect of lovastatin (mevinolin) after tube formation. (a)-(c) After tube formation, lovastatin treatment induced the degradation of newly formed capillary tubes (arrows). (a) Before Lovastatin treatment; (b) after 24 hours; (c) after 48 hours; rat aortic explant (*). Scale bar $=200 \mu \mathrm{m}$.

shape (Figure 10(a)), although some polygonal cells with cell processes maintained their morphology (Figure 10(b)).

3.7. Effect of Endothelial Cell Scraping. For the everted aorta with intact epithelium, spindle-shaped cells migrated into the collagen gels, and capillary tube formation occurred in a similar manner (Figure 11(a)). After endothelial cell scraping, spindle-shaped cells migrated into the collagen gels from the everted aorta, even without the presence of epithelial cells. However, capillary tube formation did not occur (Figure 11(b)).

\section{Discussion}

Recently, LS-7 (amino acid sequence: LQNAPRS), which is a specific binding peptide that targets mouse CD133, was screened and identified for the first time using phage-displayed peptide library technology [24]. However, the biological function of CD133 remains unclear. CD133 expression is not restricted to the neuroepithelial and hematopoietic stem and progenitor cells in which it was originally observed; it also extends to several epithelial and nonepithelial cell types. CD133 is also widely used as a marker for cancer stem cells (CSCs) in many different types of solid tumors including colon [25, 26], brain [27, 28], skin [29], pancreatic [30], liver [31-33], and prostate [34] tumors. Wang et al. [7] and RicciVitiani et al. [35] presented evidence that tumor-derived endothelial cells arise from tumor stem-like cells. Wang et al. [7] found that a glioblastoma cell population that could differentiate into endothelial cells and form intracellular vacuolar structures in collagen gels was enriched in cells expressing CD133. Although the possibility of endothelial differentiation of tumor cells has been suggested in lymphoma, myeloma, chronic myeloid leukemia, breast cancer, and neuroblastoma [36-40], the angiogenic activity of CSCs has not been investigated in other types of tumors. Because glioblastoma is one of the most vascular-rich tumors, further investigation is needed to evaluate the differentiation of CSCs into endothelial cells.

In the present study, we demonstrated that CD133-positive cells were present in the newly formed capillary tubes. Wang et al. [7] suggested that the differentiation of CSCs into endothelial cells may be mediated by signaling pathways 


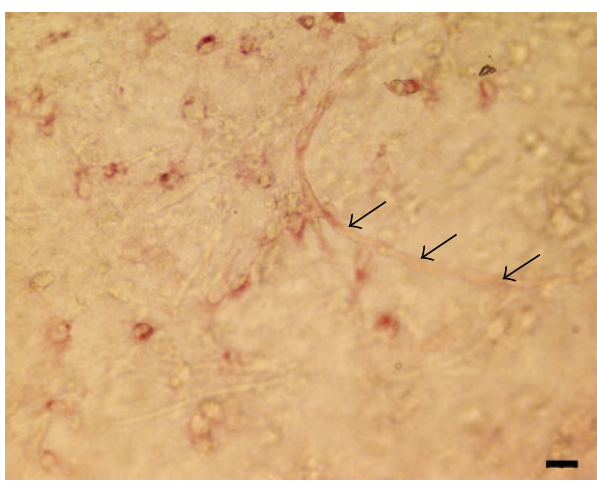

(a)

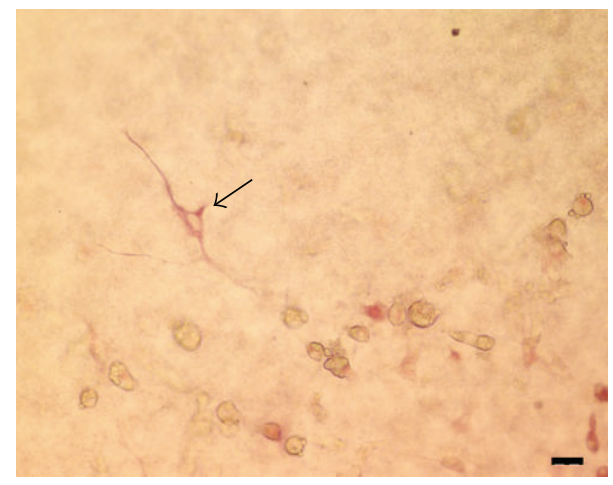

(b)

Figure 10: (a) Cell-cell adhesion was diminished, and many CD133-positive cells adopted a round morphology. Arrows indicated the degradation of capillary tubes. Scale bar $=20 \mu \mathrm{m}$. (b) Some polygonal cells with cell processes maintained their morphology (arrow). Scale bar $=20 \mu \mathrm{m}$.

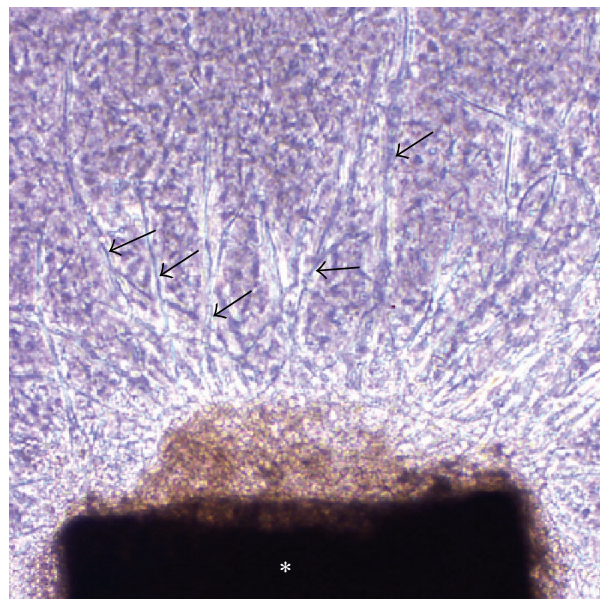

(a)

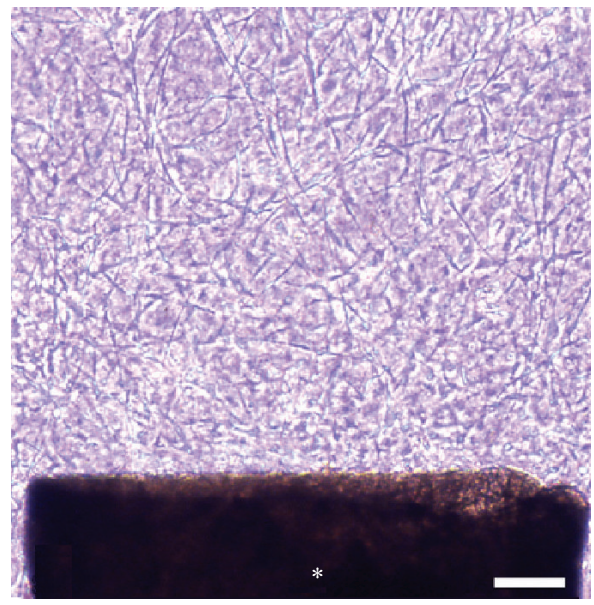

(b)

Figure 11: (a) A control culture from the rat aorta explant. Many capillary tubes (arrows) are present. (b) A deendothelialized rat aortic explant that has spindle-shaped cells migrating into collagen gels. However, capillary tube formation did not occur. Rat aortic explant $(*)$, Giemsa stain. Scale bar $=200 \mu \mathrm{m}$.

involving two proteins: vascular endothelial growth factor (VEGF) and notch. Wang et al. [7] proposed that notch regulates the initial differentiation of cancer stem cells to endothelial progenitor cells, whereas VEGF selectively affects the differentiation of endothelial progenitors to tumorderived endothelial cells. We showed that the leading edge of the capillary tube was strongly positive for CD133, which suggests that CD133-positive cells are involved in the elongation and/or branching of capillary tubes. Mature endothelial cells do not express CD133. CD133 is expressed by endothelial precursors and rapidly lost upon differentiation into mature endothelial cells [41]. It is therefore likely that the newly formed capillary tubes that consist of CD133-positive cells are immature. Soda et al. [42] reported that hypoxia-inducible factor-1 (HIF-1) is an important enhancer of EC differentiation of tumor cells and that the formation of tumor-derived endothelial cells is independent of VEGF. Hypoxic conditions may have thus resulted in the formation of CD133-positive capillary tubes at the bottom of the collagen gels.
The present study showed that CD133-positive cells were also present within aortic explants. Zengin et al. [43] reported the existence of endothelial precursor and stem cells in a distinct zone of the vascular wall that are capable to differentiate into mature endothelial cells, hematopoietic cells, and local immune cells such as macrophages. This zone has been identified to be loculated between the smooth muscle and the adventitial layer of the adult human vascular wall. Progenitor cells isolated from the adventitia of both murine and human blood vessels have the potential to form endothelial cells, mural cells, osteogenic cells, and adipocytes. These progenitors appear to cluster at or near the border zone between the outer media and inner adventitia [44]. After scraping the epithelium, spindle-shaped cells migrated into the collagen gels from both ends of the everted aorta. However, capillary tube formation did not occur. In this aortic culture, it is likely that the primary source of newly formed capillary tubes is the intimal endothelial cells. Nicosia [12] also noted that rat carotid artery explants failed to generate an angiogenic 
response when completely deendothelialized with a balloon catheter, whereas control arteries with an intact intimal endothelium produced microvessels from their resected ends. At the present time, it is unclear whether the primary source of newly formed capillary tubes is derived from the distinct zone between the smooth muscle and the adventitial layer or from the intimal endothelial cell layer. Further studies are needed to clarify these issues.

The present study also showed that lovastatin strongly inhibited cell migration from the aortic explant. The mechanism of inhibition of cell migration is considered as follows. Lovastatin is a potent inhibitor of hydroxymethylglutarylcoenzyme A (HMG-CoA) reductase. The inhibition of HMG-CoA reductase, which is involved in lipid metabolism, causes the lipids necessary for the normal membrane functioning to become defective, and further impairment is seen in their adhesive properties. The adhesion of lipids is mediated through integrins, which are necessary for cell motility and migration through the extracellular matrix in the process of invasion [22]. Clinical data indicate that statin-treated patients have diminished intraplaque angiogenesis [45], which suggests that statins have angiostatic effects in vivo. It is also intriguing that statins have been reported to reduce the growth and spread of many cancers $[46,47]$, which may be related to inhibition of angiogenesis [48]. The present study also showed that lovastatin cased the abrogation of cell-cell adhesion and degradation of capillary tubes. Khaidakov et al. [48] suggested that statins through VE-cadherin stimulation modulate cell-cell adhesion and diminish the ability of cells to proliferate and migrate. Recently, Koyama-Nasu et al. [49] reported that CD133 interacts with plakoglobin (also known as c-catenin), a desmosomal linker protein. They further demonstrate that knockdown of CD133 by RNA interference (RNAi) results in the downregulation of desmoglein-2, a desmosomal cadherin, and abrogates cell-cell adhesion and tumorigenicity of clear cell carcinoma of the ovary stem cells. In addition, we reported that the cholesterol chelating agent, methyl- $\beta$-cyclodextrin, diminished cell adhesion by decreasing desmosomes and intercellular digitations. A decrease in the cholesterol level may perturb CD133 membrane localization [50]. Modulation of cell-cell adhesion may help to explain the degradation of the capillary tubes. The present study thus provides insight into the function of CD133 during angiogenesis and provides an explanation for the antiangiogenic effect of statins.

\section{Conclusions}

The CD133-positive cell population has the capacity to form capillary tubes. The present study provides a useful method for determining the function of CD133 during angiogenesis.

\section{Conflict of Interests}

The authors report no conflict of interests concerning the materials or methods used in this study or the findings specified in this paper.

\section{Acknowledgment}

This work was supported in part by JSPS KAKENHI Grant nos. 22591985 and 25462779.

\section{References}

[1] S. Miraglia, W. Godfrey, A. H. Yin et al., "A novel fivetransmembrane hematopoietic stem cell antigen: isolation, characterization, and molecular cloning," Blood, vol. 90, no. 12, pp. 5013-5021, 1997.

[2] A. Weigmann, D. Corbeil, A. Hellwig, and W. B. Huttner, "Prominin, a novel microvilli-specific polytopic membrane protein of the apical surface of epithelial cells, is targeted to plasmalemmal protrusions of non-epithelial cells," Proceedings of the National Academy of Sciences of the United States of America, vol. 94, no. 23, pp. 12425-12430, 1997.

[3] U. M. Gehling, S. Ergün, U. Schumacher et al., "In vitro differentiation of endothelial cells from AC133-positive progenitor cells," Blood, vol. 95, no. 10, pp. 3106-3112, 2000.

[4] G. Invernici, C. Emanueli, P. Madeddu et al., "Human fetal aorta contains vascular progenitor cells capable of inducing vasculogenesis, angiogenesis, and myogenesis in vitro and in a murine model of peripheral ischemia," The American Journal of Pathology, vol. 170, no. 6, pp. 1879-1892, 2007.

[5] L. S. Barcelos, C. Duplaa, N. Kränkel et al., "Human CD133+ progenitor cells promote the healing of diabetic ischemic ulcers by paracrine stimulation of angiogenesis and activation of Wnt signaling," Circulation Research, vol. 104, no. 9, pp. 1095-1102, 2009.

[6] V. L. Bautch, “Tumour stem cells switch sides," Nature, vol. 468, no. 7325, pp. 770-771, 2010.

[7] R. Wang, K. Chadalavada, J. Wilshire et al., "Glioblastoma stemlike cells give rise to tumour endothelium," Nature, vol. 468, no. 7325, pp. 829-833, 2010.

[8] R. Montesano, P. Mouron, and L. Orci, "Vascular outgrowths from tissue explants embedded in fibrin or collagen gels: a simple in vitro model of angiogenesis," Cell Biology International Reports, vol. 9, no. 10, pp. 869-875, 1985.

[9] R. F. Nicosia and A. Ottinetti, "Modulation of microvascular growth and morphogenesis by reconstituted basement membrane gel in three-dimensional cultures of rat aorta: a comparative study of angiogenesis in Matrigel, collagen, fibrin, and plasma clot," In Vitro Cellular and Developmental Biology, vol. 26, no. 2, pp. 119-128, 1990.

[10] M. Mori, Y. Sadahira, S. Kawasaki, T. Hayashi, K. Notohara, and M. Awai, "Capillary growth from reversed rat aortic segments cultured in collagen gel," Acta Pathologica Japonica, vol. 38, no. 12, pp. 1503-1512, 1988.

[11] R. F. Nicosia and A. Ottinetti, "Growth of microvessels in serum-free matrix culture of rat aorta. A quantitative assay of angiogenesis in vitro," Laboratory Investigation, vol. 63, no. 1, pp. 115-122, 1990.

[12] R. F. Nicosia, "The aortic ring model of angiogenesis: a quarter century of search and discovery," Journal of Cellular and Molecular Medicine, vol. 13, no. 10, pp. 4113-4136, 2009.

[13] A. C. Aplin, W. H. Zhu, E. Fogel, and R. F. Nicosia, "Vascular regression and survival are differentially regulated by MT1MMP and TIMPs in the aortic ring model of angiogenesis," The American Journal of Physiology, vol. 297, no. 2, pp. C471-C480, 2009. 
[14] M. Akita, E. Murata, H.-J. Merker, and K. Kaneko, "Morphology of capillary-like structures in a three-dimensional aorta/collagen gel culture," Annals of Anatomy, vol. 179, no. 2, pp. 127-136, 1997.

[15] M. Akita, E. Murata, H.-J. Merker, and K. Kaneko, "Formation of new capillary-like tubes in a three-dimensional in vitro model (Aorta/Collagen Gel)," Annals of Anatomy, vol. 179, no. 2, pp. 137-147, 1997.

[16] T. Nagatoro, K. Fujita, E. Murata, and M. Akita, "Angiogenesis and Fibroblast Growth Factors (FGFs) in a three-dimensional collagen gel culture," Okajimas Folia Anatomica Japonica, vol. 80, no. 1, pp. 7-14, 2003.

[17] K. Fujita, Y. Asami, E. Murata, M. Akita, and K. Kaneko, "Effects of thalidomide, cytochrome P-450 and TNF- $\alpha$ on angiogenesis in a three-dimensional collagen gel-culture," Okajimas Folia Anatomica Japonica, vol. 79, no. 4, pp. 101-106, 2002.

[18] K. Fujita, Y. Asami, K. Tanaka, M. Akita, and H.-J. Merker, "Anti-angiogenic effects of thalidomide: expression of apoptosis-inducible active-caspase- 3 in a three-dimensional collagen gel culture of aorta," Histochemistry and Cell Biology, vol. 122, no. 1, pp. 27-33, 2004.

[19] K. Fujita, K. Komatsu, K. Tanaka et al., "An in vitro model for studying vascular injury after laser microdissection," Histochemistry and Cell Biology, vol. 125, no. 5, pp. 509-514, 2006.

[20] M. Akita and K. Fujita, "DNA micro-array gene expression profiling of angiogenesis in collagen gel culture," Clinical Medicine, vol. 2, pp. 49-57, 2008.

[21] S. Hoffmann, C. Spee, T. Murata, J. Z. Cui, S. J. Ryan, and D. R. Hinton, "Rapid isolation of choriocapillary endothelial cells by Lycopersicon esculentum-coated Dynabeads," Graefe's Archive for Clinical and Experimental Ophthalmology, vol. 236, no. 10, pp. 779-784, 1998.

[22] I. Depasquale and D. N. Wheatley, "Action of Lovastatin (Mevinolin) on an in vitro model of angiogenesis and its co-culture with malignant melanoma cell lines," Cancer Cell International, vol. 6, article 9, 2006.

[23] R. F. Nicosia, E. Bonanno, and S. Villaschi, "Large-vessel endothelium switches to a microvascular phenotype during angiogenesis in collagen gel culture of rat aorta," Atherosclerosis, vol. 95, no. 2-3, pp. 191-199, 1992.

[24] J. Sun, C. Zhang, G. Liu et al., "A novel mouse CD133 bindingpeptide screened by phage display inhibits cancer cell motility in vitro," Clinical and Experimental Metastasis, vol. 29, no. 3, pp. 185-196, 2012.

[25] C. A. O’Brien, A. Pollett, S. Gallinger, and J. E. Dick, "A human colon cancer cell capable of initiating tumour growth in immunodeficient mice," Nature, vol. 445, no. 7123, pp. 106-110, 2007.

[26] L. Ricci-Vitiani, D. G. Lombardi, E. Pilozzi et al., "Identification and expansion of human colon-cancer-initiating cells," Nature, vol. 445, no. 7123, pp. 111-115, 2007.

[27] S. K. Singh, C. Hawkins, I. D. Clarke et al., "Identification of human brain tumour initiating cells," Nature, vol. 432, no. 7015, pp. 396-401, 2004.

[28] G. Liu, X. Yuan, Z. Zeng et al., "Analysis of gene expression and chemoresistance of CD133+ cancer stem cells in glioblastoma," Molecular Cancer, vol. 5, article 67, 2006.

[29] E. Monzani, F. Facchetti, E. Galmozzi et al., "Melanoma contains CD133 and ABCG2 positive cells with enhanced tumourigenic potential," The European Journal of Cancer, vol. 43, no. 5, pp. 935-946, 2007.
[30] M. Olempska, P. A. Eisenach, O. Ammerpohl, H. Ungefroren, F. Fandrich, and H. Kalthoff, "Detection of tumor stem cell markers in pancreatic carcinoma cell lines," Hepatobiliary and Pancreatic Diseases International, vol. 6, no. 1, pp. 92-97, 2007.

[31] A. Suetsugu, M. Nagaki, H. Aoki, T. Motohashi, T. Kunisada, and H. Moriwaki, "Characterization of CD133+ hepatocellular carcinoma cells as cancer stem/progenitor cells," Biochemical and Biophysical Research Communications, vol. 351, no. 4, pp. 820-824, 2006.

[32] S. Yin, J. Li, C. Hu et al., "CD133 positive hepatocellular carcinoma cells possess high capacity for tumorigenicity," International Journal of Cancer, vol. 120, no. 7, pp. 1444-1450, 2007.

[33] S. Hayashi, K. Fujita, S. Matsumoto, M. Akita, and A. Satomi, "Isolation and identification of cancer stem cells from a side population of a human hepatoblastoma cell line, HuH-6 clone5," Pediatric Surgery International, vol. 27, no. 1, pp. 9-16, 2011.

[34] A. T. Collins, P. A. Berry, C. Hyde, M. J. Stower, and N. J. Maitland, "Prospective identification of tumorigenic prostate cancer stem cells," Cancer Research, vol. 65, no. 23, pp. 1094610951, 2005.

[35] L. Ricci-Vitiani, R. Pallini, M. Biffoni et al., "Tumour vascularization via endothelial differentiation of glioblastoma stem-like cells," Nature, vol. 468, no. 7325, pp. 824-828, 2010.

[36] E. Gunsilius, H.-C. Duba, A. L. Petzer et al., "Evidence from a leukaemia model for maintenance of vascular endothelium by bone-marrow-derived endothelial cells," The Lancet, vol. 355, no. 9216, pp. 1688-1691, 2000.

[37] B. Streubel, A. Chott, D. Huber et al., "Lymphoma-specific genetic aberrations in microvascular endothelial cells in B-cell lymphomas," The New England Journal of Medicine, vol. 351, no. 3, pp. 250-259, 2004.

[38] G. M. Rigolin, C. Fraulini, M. Ciccone et al., "Neoplastic circulating endothelial cells in multiple myeloma with 13q14 deletion,” Blood, vol. 107, no. 6, pp. 2531-2535, 2006.

[39] A. Pezzolo, F. Parodi, M. V. Corrias, R. Cinti, C. Gambini, and V. Pistoia, "Tumor origin of endothelial cells in human neuroblastoma," Journal of Clinical Oncology, vol. 25, no. 4, pp. 376383, 2007.

[40] B. Bussolati, C. Grange, A. Sapino, and G. Camussi, "Endothelial cell differentiation of human breast tumour stem/progenitor cells," Journal of Cellular and Molecular Medicine, vol. 13, no. 2, pp. 309-319, 2009.

[41] M. Peichev, A. J. Naiyer, D. Pereira et al., "Expression of VEGFR2 and AC133 by circulating human CD34+ cells identifies a population of functional endothelial precursors," Blood, vol. 95, no. 3, pp. 952-958, 2000.

[42] Y. Soda, T. Marumoto, D. Friedmann-Morvinski et al., “Transdifferentiation of glioblastoma cells into vascular endothelial cells," Proceedings of the National Academy of Sciences of the United States of America, vol. 108, no. 11, pp. 4274-4280, 2011.

[43] E. Zengin, F. Chalajour, U. M. Gehling et al., "Vascular wall resident progenitor cells: a source for postnatal vasculogenesis," Development, vol. 133, no. 8, pp. 1543-1551, 2006.

[44] M. W. Majesky, X. R. Dong, V. Hoglund, G. Daum, and W. M. Mahoney Jr., "The adventitia: a progenitor cell niche for the vessel wall," Cells Tissues Organs, vol. 195, no. 1-2, pp. 73-81, 2011.

[45] V. J. Karthikeyan and G. Y. H. Lip, "Statins and intra-plaque angiogenesis in carotid artery disease," Atherosclerosis, vol. 192, no. 2, pp. 455-456, 2007.

[46] J. N. Poynter, S. B. Gruber, P. D. R. Higgins et al., "Statins and the risk of colorectal cancer," The New England Journal of Medicine, vol. 352, no. 21, pp. 2184-2192, 2005. 
[47] S. Friis, A. H. Poulsen, S. P. Johnsen et al., "Cancer risk among statin users: a population-based cohort study," International Journal of Cancer, vol. 114, no. 4, pp. 643-647, 2005.

[48] M. Khaidakov, W. Wang, J. A. Khan, B.-Y. Kang, P. L. Hermonat, and J. L. Mehta, "Statins and angiogenesis: is it about connections?" Biochemical and Biophysical Research Communications, vol. 387, no. 3, pp. 543-547, 2009.

[49] R. Koyama-Nasu, R. Takahashi, S. Yanagida et al., The cancer stem cell marker CD133 interacts with plakoglobin and controls desmoglein-2 protein levels, vol. 8, no. 1, Article ID e53710, 2013.

[50] M. Akita, K. Tanaka, N. Murai et al., "Detection of CD133 (prominin-1) in a human hepatoblastoma cell line (HuH-6 clone 5)," Microscopy Research and Technique, 2013 http://onlinelibrary.wiley.com/doi/10.1002/jemt.22237/abstract. 

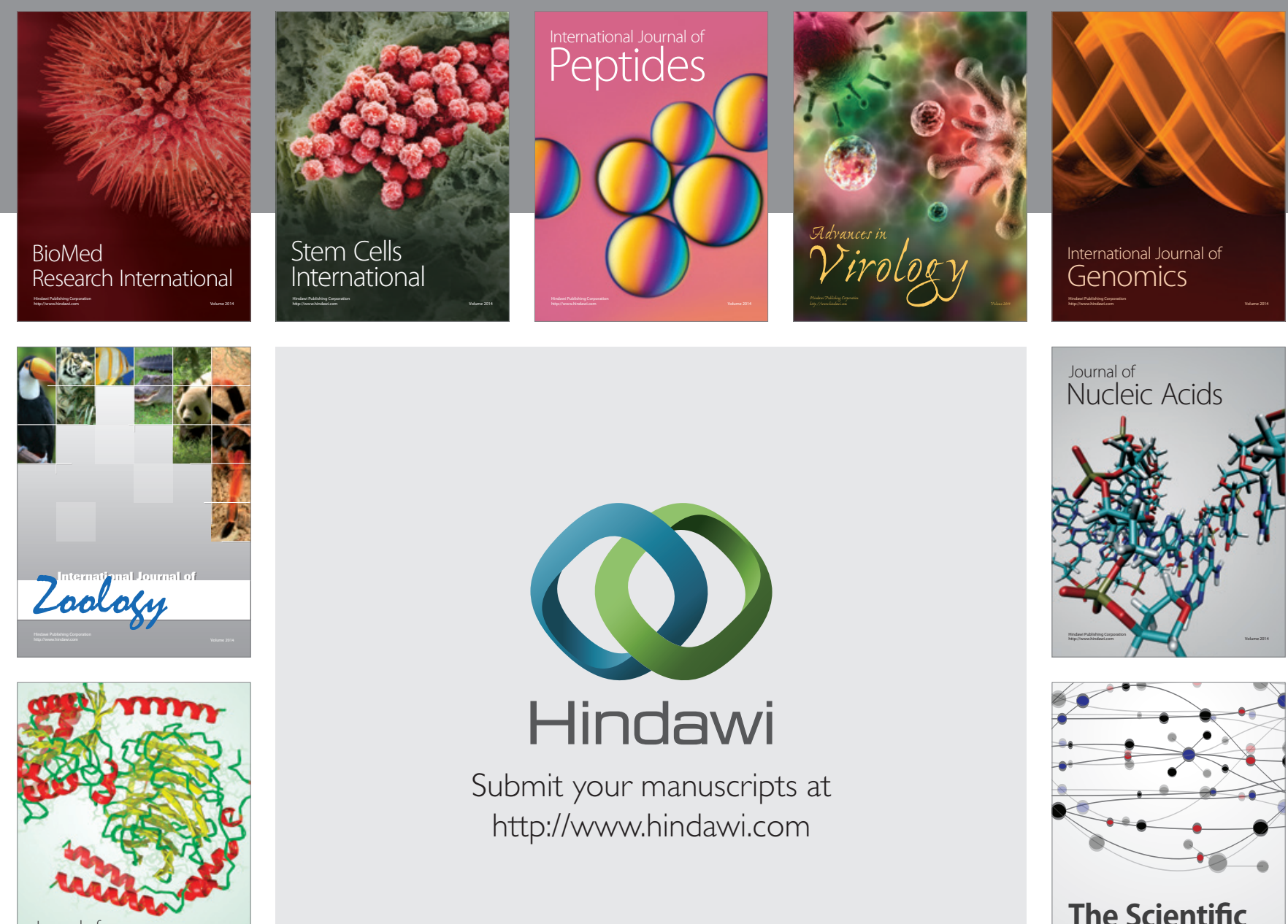

Submit your manuscripts at

http://www.hindawi.com

Journal of
Signal Transduction
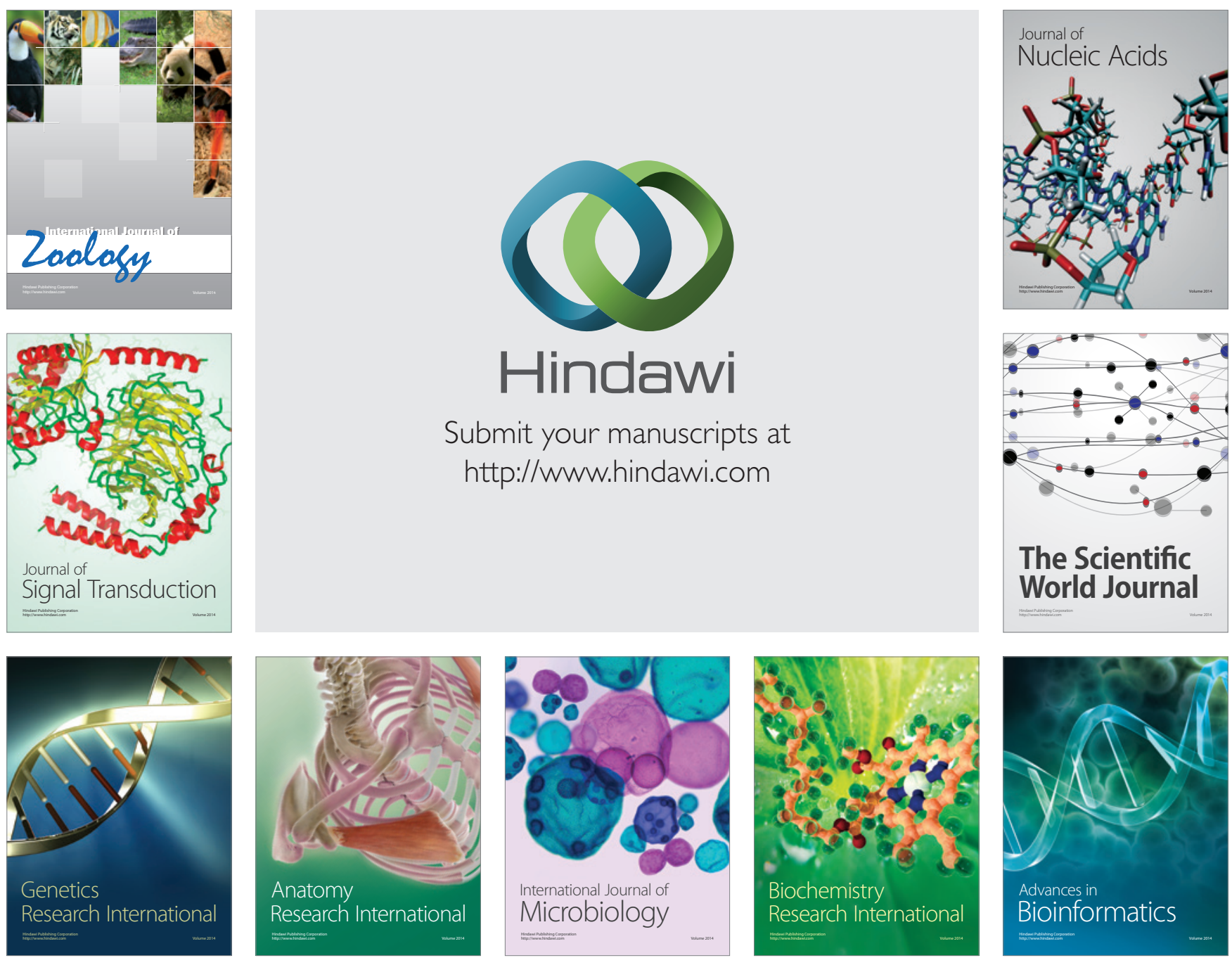

The Scientific World Journal
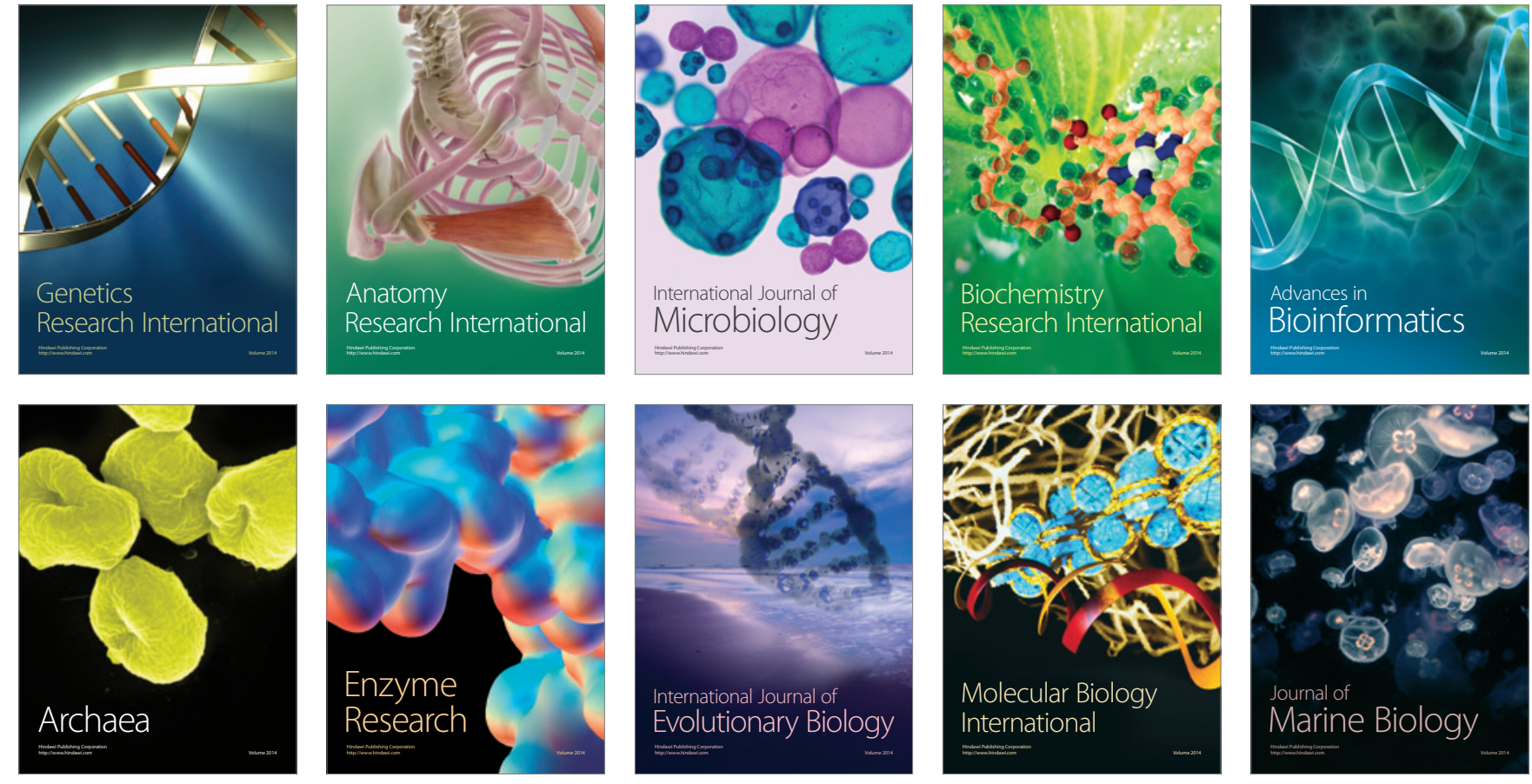